\title{
Solución numérica de problemas de valores en la frontera con alta exactitud por métodos espectrales
}

\author{
A. Camacho-Galván (1), B. Guardián-Soto (2) y M. Rodríguez-Green (1) \\ (1) Facultad de Ingeniería, UNAM y (2) ESIME Culhuacán, IPN \\ E-mail: abelcgmx@yahoo.com.mxy bdguardian@ipn.mx
}

(recibido: septiembre de 2004; aceptado: febrero de 2005)

\begin{abstract}
Resumen
Entre la variedad de métodos disponibles para resolver problemas de valores en la frontera se cuentan los métodos espectrales, donde la solución $u(x, t)$ de un cierto problema de valores en la frontera (dependiente de una vari able espacial $\mathrm{x}$ y de una variable temporal), se aproxima por una suma finita $v(x, t)=\sum_{k=0}^{N} a_{k}(t) \phi_{k}(x)$. Para este fin se debe, por una parte, seleccionar la clase de las funciones $\phi_{k}(x), k=1, \ldots, N$, y por otra, prefijar los algoritmos adecuados para el desarrollo de los coeficientes $a_{k}$.
\end{abstract}

Descriptores: Problemas de valores en la frontera, ecuaciones diferenciales en derivadas parciales, procesamiento paralelo, métodos pseudoespectrales

\begin{abstract}
A bstract
A mong the meth ods available for theso lu tion of bound ary prob lems wemay find the so-called spectral meth ods, in which thesolution $u(x, t)$ (depend ing upon a space likevari ablex and a time likevariable) is ap proxi mated by a fi itesumv $(x, t)=\sum_{k=0}^{N} a_{k}(t) \phi_{k}(x)$. In or der to have this form, on one hand, a class of functions $\phi_{\mathrm{k}}(\mathrm{x}), \mathrm{k}=1, \ldots, N$, has to bese lected, and on theother, thead equateal go rithms for theex pan sion of the co ef fi cients ak must becho sen.
\end{abstract}

Keywords: Numerical techniques, finite difference methods, collocation methods, Chebyshev approximation, Gauss-Lobatto points, Gauss-Radau points.

\section{Introducción}

Aun cuando existe una amplia variedad de métodos para resolver problemas de valores en la frontera, tales como los métodos en diferencias finitas, el método del elemento fini to, el método del volumen fini to, etc., a la fecha, cuando se busca aproximar la solución numérica de un problema con alta exactitud, son los métodos espectrales los recursos favorecidos.

Estos se inician con el método de Fourier aplicado a las ecuaciones diferenciales, donde la solución se representa mediante una serie truncada, siendo las incógnitas los coeficientes de los términos que la integran.
Desde épocas que anteceden a la aparición de las computadoras, los métodos espectrales se desarrollaron y obtuvieron amplio reconocimiento, pero al inicio de la mecánica de fluidos computacional, cuando se atacaron problemas alineales, aquellos mostraron severas limitaciones.

La aplicación ineficiente de las series de Fourier truncadas, provocada por la gran cantidad de términos por estimar, desembocó en beneficio de los métodos en diferencias y del método del elemento finito; en cuanto a éstos, en el estudio de problemas de alta complejidad se sabe cómo su deficiente capacidad de discretización constituye un severo obstáculo para el logro de una adecuada aproximación numérica de la solución. 
El resurgimiento actual de los métodos espectrales se debe especialmente a:

I. La potencia de los modernos procesadores.

II. La eficiencia de la transformada rápida de

Fourier en la evaluación de sumas.

III. La posibilidad de expresar la solución aproximada en términos tensoriales.

Pioneros de esta etapa fueron Kreiss (1968) y Orzag (1969); por otro lado, Gottlieb (1978); (1987), mostró, en cuanto la obtención de una representación cómoda de las funciones en el espacio físico, la utilidad de posicionar los nodos en la malla mediante la cuadratura gaussiana.

Si bien, el método de Fourier funciona con propiedad en problemas periódicos, cuando se abordan problemas aperiódicos por causa del fenómeno de Gibbs, presenta serias deficiencias. En este caso, otros tipos de polinomios ortogonales constituyen una afortunada alternativa.

De estas sencillas observaciones, se obtienen novedosos procedimientos: las diferenciaciones se ejecutan en el espacio espectral mientras los productos se realizan en el espacio físico, la conexión entre ambos se obtiene vía la transformada rápida de Fourier.

Al tratar problemas diferenciales, el carácter global de los métodos espectrales generalmente favorece la exactitud de los resultados. Por otro lado, la fuerte interrelación que se muestra entre los valores en distintos puntos, puede perturbar significativamente los resultados.

Estas dificultades pueden so slayarse aplicando técnicas de filtrado (Gottlieb, 1997), métodos de descomposición del dominio (Guillard et al., 1992) o técnicas de sustracción de singularidades (Botella, 2001a y 2001b). Por lo general, las matrices obtenidas no resultan ni simétricas ni antisimétricas y suelen quedar mal condicionadas.

A pesar de estos inconvenientes, cuando se busca representar con alta exactitud las soluciones de problemas asociados a funciones suaves, se recomienda la aplicación de los méto dos espectrales.

\section{Métodos espectrales}

\section{Clasificación y polinomios ortogonales útiles}

En esta sección se describen algunas de las características principales más comunes a los métodos espectrales. Éstos se clasifican en dos grandes grupos:

A. Métodos interpolantes o pseudoespectrales, que demandan la satisfacción exacta de la ecuación diferencial en un cierto conjunto de puntos de la malla. Generalmente, la solución se busca en la forma de una serie truncada.

B. Métodos no-interpolantes, donde la función incógnita se aproxima por una serie truncada y los coeficientes se evalúan mediante multiplicaciones por las funciones base y algoritmos de integración.

A los primeros también se les conoce como métodos de colocación, ejemplos de los segundos son el método de Galerkin y el método Tau de Lanczos.

A su vez, son las condiciones de frontera las que determinan las funciones base; por ejemplo, si las condiciones requieren soluciones periódicas, se recurre a las series de Fourier, donde las funciones sen(?) y $\cos ($ ?) son las adecuadas.

Los tipos de funciones base recomendados para distintos casos, se describen a continuación:

I. Condiciones de frontera periódicas, se aplican series de Fourier.

II. Condiciones de frontera aperiódicas en intervalos acotados, se usan polinomios de Chebyshev o polinomios de Legendre.

III. Condiciones de frontera en intervalos semi-infinitos, se recurre a los polinomios racionales de Chebyshev TL o polinomios de Legendre.

IV. Condiciones de frontera en intervalos infinitos, se usan polinomios racionales de Chebyshev TB o polinomios de Hermite. 


\section{Esquema de los métodos espectrales}

Considérese ahora el operador diferencial $L$ definido en la región Ocon frontera $\partial \Omega \subseteq R^{\text {n }}$ y sea $B$ una función definida en $\partial \Omega$ estipulada por las condiciones de frontera de Dirichlet, Cauchy 0 Robin.

Se busca la solución numérica del problema:

Problema 1

$$
\begin{aligned}
& L(u)=f \text { en } \Omega, \\
& B(u)=g \text { en } \partial \Omega
\end{aligned}
$$

En general, los métodos espectrales comprenden los siguientes puntos:

1. En el dominio $\Omega \subset R^{\mathrm{n}}$ se toma una partición compuesta por subdominios $\Omega_{\mathrm{m}}, \mathrm{m}=1, \ldots N$. Es decir:

$$
\Omega=\bigcup_{m=1}^{N} \Omega_{m}, \Omega_{i} \cap \Omega_{j}=\phi, \text { si } \neq j .
$$

La figura 1 ilustra la partición de un dominio acotado $\Omega \subset R^{2}$ con frontera:

$\partial \Omega ; \Omega=\Omega_{1} \cup \Omega_{2} \cup \Omega_{3} \cup \Omega_{4} \cup \Omega_{5} \cup \Omega_{6} \cup \Omega_{7}$.

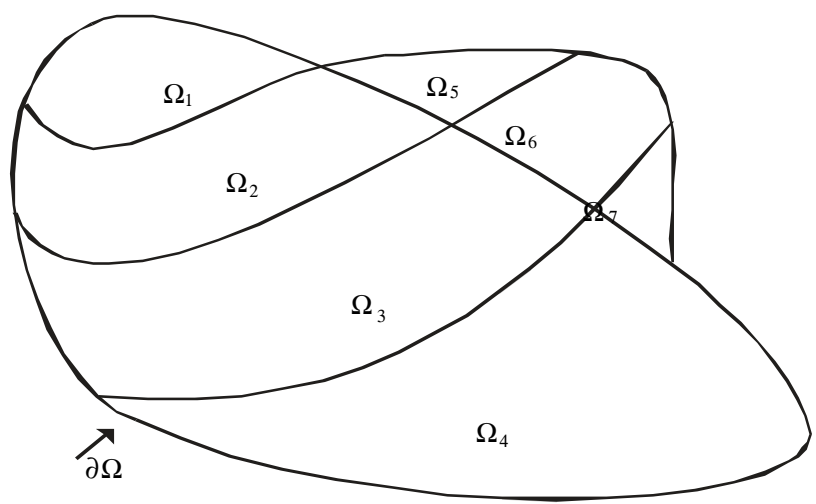

Figura 1. Ejemplo de dominio $\Omega \subset R^{2}$ acotado

2. Cada subdominio $\Omega_{m}$ es la transformación suave $T_{\mathrm{m}}$ de un elemento tipo $\hat{\Omega}$.

Por su parte, el elemento de referencia $\hat{\Omega}$ es el producto de los intervalos $\hat{I}_{\mathrm{k}}$ del eje real, es decir:

$$
\Omega=\prod_{\mathrm{k}=1}^{\mathrm{n}} \hat{I}_{\mathrm{k}} .
$$

En la figura 2 se muestra al subdominio $\Omega_{\mathrm{m}}$, en el plano $x-y$, obtenido bajo la transformación $T_{m}$ del elemento tipo

$$
\hat{\Omega}=[-1,1] \times[-1,1]
$$

en el plano $\xi-\eta$.

El espacio $\mathrm{S}_{\mathrm{N}}(\hat{\Omega})$ de las funciones discretas en $\Omega$ se obtiene con base en las funciones discretas definidas en $\hat{\Omega}$.

Sea el isomorfismo topológico lineal:

$$
F: S_{\mathrm{N}}\left(R^{\mathrm{n}}\right) \rightarrow S_{\mathrm{N}}\left(R^{\mathrm{n}}\right)
$$

tal que,

$$
S_{\mathrm{N}}\left(\Omega_{\mathrm{m}}\right)=F_{m}\left(S_{\mathrm{N}}(\hat{\Omega})\right) \quad \mathrm{m}=1,2, \ldots, M
$$

y de aquí

$$
F_{m}\left(S_{\mathrm{N}}(\hat{\Omega})\right)=\left\{\hat{a}_{N}\left(F_{m}^{-1}(x) \mid \mathfrak{a}_{N} \in S_{\mathrm{N}}(\hat{\Omega})\right)\right\}
$$

siendo en consecuencia

$$
S_{N}(\Omega)=\left\{\mathrm{u}_{N}: \Omega \rightarrow R \mid \mathrm{u}_{N \mid \Omega_{m}} \in S_{\mathrm{N}}\left(\Omega_{\mathrm{m}}\right), \forall \mathrm{m}\right\}
$$

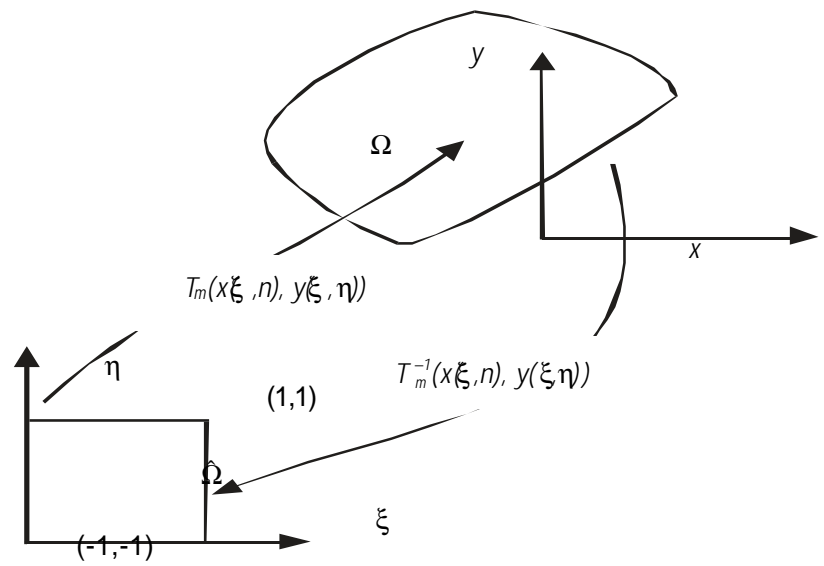

Figura 2. Subdominio $\Omega_{\mathrm{m}}$ obtenido como imagen del elemento tipo $\hat{\Omega}$

4. El espacio discreto posee estructura tensorial 


$$
S_{N}(\hat{\Omega})=\bigotimes_{i=1}^{n} S_{N_{i}}\left(\hat{I}_{\mathrm{i}}\right)
$$

5. Exactitud espectral. Se ha demostrado por (Gottlieb y Orzag, 1978); (Canuto et al., 1988) y (Mercier, 1989) como el espacio discreto $S_{N}(\hat{I})$ posee la denominada propiedad de exactitud expectral; es decir, si $\|\hat{v}\|$ es una norma $L^{2}$ en $\hat{I}$, luego para enteros $s>0$, existe una constante $C_{\mathrm{s}}$ tal que

$$
\inf _{\left.\mathfrak{u}_{N} \in S_{N} \hat{I}\right)}\left\|\hat{\mathrm{u}}-\hat{\mathrm{u}}_{N}\right\| \leq C_{S} N^{-s} \sum_{\mathrm{i}=0}^{s}\left\|D^{l} \hat{\mathrm{u}}\right\|
$$

para toda $\hat{a}$, aquí $D^{\prime} \hat{a}=\frac{d^{\prime} \hat{u}}{d \hat{x}^{\prime}}$

En otras palabras, cuando $N \rightarrow \infty$, el error para û en $S_{N}(\hat{I})$ depende de la regularidad de la función û, luego para funciones infinitamente diferenciables el error decrece con razón mayor que cualquier potencia de $N^{-1}$, es decir, el orden de aproximación es infinito.

El espacio físico y el espacio en la frecuencia

Considérese el conjunto $\left\{\phi_{k}(x)\right\}$ definido en el intervalo $\hat{I}$, de funciones trigonométricas o polinomiales de orden $k$, ortogonales respecto a una cierta función de peso $\mathrm{w}(\mathrm{x})$, es decir,

$$
\left(\phi_{m}, \phi_{n}\right)=c_{m} \delta_{m},
$$

donde $c_{m}$ es una constante, $\delta_{m}$ es la función delta de Kroneckery

$$
\left(\phi_{m}, \phi_{n}\right)=\int_{\Gamma} \phi_{m}(x) \phi_{n}(x) \omega(x)
$$

La representación en el espacio en la frecuencia es generada por las funciones $\phi_{k}$ :

y aquí

$$
S_{N}=\operatorname{gen}\left\{\phi_{\mathrm{k}}, \mathrm{k}=0, \ldots, N\right\} \text {, }
$$

$$
\hat{\mathrm{u}}_{N} \in S_{N}(\hat{I}) \Leftrightarrow \hat{\mathrm{u}}_{N}(\mathrm{x})=\sum_{k=0}^{N} \hat{\mathrm{u}}_{\mathrm{k}} \phi_{\mathrm{k}}(\mathrm{x})
$$

Recíprocamente, la representación en el espacio físico se obtiene como se indica a continuación:
En el conjunto $\hat{I} \cup \partial \hat{I}$ mediante cuadratura Gaussiana, se fijan $N+1$ puntos distintos

$$
\mathrm{X}_{0}<\mathrm{x}_{1}<\ldots \mathrm{x}_{N-1}<\mathrm{x}_{N}
$$

y con base en estos puntos se construyen las bases de Lagrange

$$
I_{j}(x) \prod_{k=0, k \neq j}^{N} \frac{x-x_{k}}{x_{k}-x_{j}}
$$

mismas que verifican las condiciones

$$
\mathrm{I}_{j}\left(\mathrm{x}_{\mathrm{i}}\right)=\delta_{\mathrm{ij}}, 0 \leq \mathrm{i}, \mathrm{j} \leq \mathrm{N}
$$

Luego:

$$
\hat{\mathrm{u}}_{N} \in S_{N}(\hat{I}) \Leftrightarrow \hat{\mathrm{u}}_{N}(\mathrm{x})=\sum_{j=0}^{N} u_{j} \mathrm{l}_{\mathrm{j}}(\mathrm{x})
$$

donde $u_{j}=\hat{u}_{N}\left(x_{j}\right)$

Existe una relación lineal $\mathbf{T}$ entre ambas representaciones, se le conoce como la "transformada discreta de Fourier", a saber

$$
\hat{\mathrm{u}}_{\mathrm{k}}=\sum_{\mathrm{j}=0}^{N} \mathrm{~T}\left\{\mathrm{u}_{\mathrm{j}}\right\}, \quad \mathrm{k}=0, \ldots, N
$$

Mientras que el proceso de transformación inverso requiere, en el caso general $\mathrm{O}\left(\mathrm{N}^{2}\right)$ operaciones, en cambio, cuando es posible aplicar la transformada de Fourier, el número disminuye a $\mathrm{O}\left(\mathrm{N} \log _{2} \mathrm{~N}\right)$ operaciones.

Obviamente, cualquier operador lineal en $\mathrm{S}_{\mathrm{N}}=\mathrm{S}_{\mathrm{N}}(\hat{I})$ puede expresarse mediante ambas representaciones, tanto en el espacio físico como en el espacio en la frecuencia.

Por otra parte, se supone que $\varsigma_{N}$ es cerrada bajo la diferenciación, si

$$
D=\frac{\mathrm{d}}{\mathrm{d} \hat{\mathrm{x}}} \text {, entonces } D S_{N} \subseteq S_{N} \text {. }
$$

\section{Polinomios de Chebyshev}

Tópicosgener ales

Los polinomios de Chebyshev del primer tipo de grado k se definen por la expresión 


$$
T_{k}(x)=\cos (k \arccos x), \quad k=0,1,2 \ldots
$$

donde $0 \leq \arccos , x \leq \pi$

Ahora bien, tomando $x=\cos$ ?, se deduce

$$
T_{k}(\cos \theta)=\cos (k \theta)
$$

y de aquí, sin mayores dificultades, se obtienen los polinomios

$$
T_{0}(\mathrm{x})=\mathrm{I}, \quad T_{1}(\mathrm{x})=\mathrm{x}
$$

Por otra parte, de la identidad trigonométrica

$$
\cos ((k+1) \theta)+\cos ((k-1) \theta)=2 \cos \theta \cos k \theta
$$

se obtiene la relación de recurrencia

$$
T_{\mathrm{k}+1}(\mathrm{x})-2 \times T_{\mathrm{k}}(\mathrm{x})+T_{\mathrm{k}-1}(\mathrm{x})=0 \text { para } \mathrm{k} \geq 1
$$

De las ecuaciones (13) y (14) se deducen los polinomios $T_{k}(x)$ para $k \geq 3$. A continuación se enlistan los cinco primeros polinomios de Chebyshev:

$$
\begin{aligned}
T_{0}(x)=1, & T_{1}(x)=x \\
T_{2}(x)=2 x^{2}-1, & T_{3}(x)=4 x^{3}-3 x \\
T_{4}(x)=8 x^{4}-8 x^{2}+1, & T_{5}(x)=16 x^{5}-20 x^{3}+5 x
\end{aligned}
$$

Seguidamente se enuncian algunas propiedades importantes de los polinomios de Chebyshev

1. Cuando $k$ es par, el polinomio $T_{k}$ es una función par.

2. Cuando $k$ es impar, el polinomio $T_{k}$ es una función impar.

3. El coeficiente mayor del polinomio $T_{k}$ cuando, $k \geq 1$ es igual a $2^{k-1}$

4. $T_{k}(x)$ tiene $k$ raíces reales en el intervalo $(-1,1)$, siendo éstas

$$
x_{i}=\cos \frac{(2 i+1) \pi}{2 k}, i=0,1, \ldots, k-1
$$

5.(a) máx $\operatorname{x\in }_{x \in[-1,1]}\left|T_{k}(x)\right|=1$

(b) $T_{k}\left(x_{i}\right)=(-1) ;$ donde $x_{i}=\cos \frac{i \pi}{k}, i=0,1, \ldots, k$
A los puntos $x_{i}$ donde $T_{k}$ alcanza los valores extremos \pm 1 se les denominan puntos de Gauss-Lobatto.

6. No existe polinomio $P_{n}(x)$ de $n$-ésimo grado con coeficiente mayor igual a la unidad, que se verifique

$$
\operatorname{máx}_{[-1, \mathrm{~g}}\left|P_{\mathrm{n}}(\mathrm{x})\right|<\operatorname{máx}_{[-1,1]}\left|T_{\mathrm{n}}(\mathrm{x})\right|=2^{1-n}
$$

7. Las derivadas de $T_{k}$ se pueden obtener mediante la siguiente relación de recurrencia

$$
T_{k}^{\prime}=\frac{d}{d \theta}(\cos k \theta) \frac{d \theta}{d x}=k \frac{\operatorname{sen} k \theta}{\operatorname{sen} \theta}
$$

y de aquí se deduce

$$
\frac{T_{k+1}^{\prime}}{k+1}-\frac{T_{k-1}^{\prime}}{k-1}=2 T_{k}
$$

8. Si se define el producto interior $L^{2}$ de los polinomios de Chebyshev $T_{k}$ y $T_{\text {I }}$ por

$$
\left(T_{\mathrm{k}}, T_{1}\right) \mathrm{W}=\int_{-1}^{1} T_{k} T_{l} \mathrm{wdx}
$$

con función de peso

$$
W=\left(1-x^{2}\right)^{-1 / 2}
$$

entonces los polinomios son ortogonales.

En particular,

$$
\left(T_{k}, T_{l}\right) \mathrm{W}=\frac{\pi}{2} \delta_{k l} c_{k}
$$

donde $\delta_{\mathrm{kl}}$ es la delta de Kronecker y

$$
c_{k}=\left\{\begin{array}{lll}
2 & \text { si } & k=0 \\
1 & \text { si } & k \geq 1
\end{array}\right.
$$

M étodo de colocación

Sea la aproximación de la función u(x) dada por la serie truncada

$$
\mathrm{u}_{N}(\mathrm{x})=\sum_{k=0}^{N} \hat{u} T_{k}(\mathrm{x}), \quad \alpha \leq \mathrm{x} \leq \beta
$$


Se introduce ahora el residual $R_{N}$ dado por

$$
R_{N}=\mathrm{U}-\mathrm{U}_{N}
$$

luego si $u_{N}(x)$ aproxima la solución de la ecuación diferencial $L u-f=0, R_{N}(\mathrm{x})=\omega_{N^{-}} \mathrm{f}$.

El método de colocación anula el residual en $N+1$ puntos denominados puntos de colocación

Por lo general, se toman como puntos de colocación los denominados puntos de Gauss-Radau o los puntos de Gauss-Lobatto (Canuto et al., 1988) y (Mercier, 1989).

> Puntos de Gauss-Radau, dados por

$$
\mathrm{x}_{\mathrm{i}}=\cos \frac{2 \pi \mathrm{i}}{2 N+1}, \mathrm{i}=0, \ldots, N
$$

> Puntos de Gauss-Lobatto dados ya por la ecuación (16), corresponden a aquellos puntos $x_{i}$ donde

$$
\left(1-\mathrm{x}^{2}\right) T_{N}^{\prime}(\mathrm{x})=0
$$

Presentan las siguientes particularidades

1. $T_{N}\left(\mathrm{x}_{\mathrm{i}}\right)=(-1)^{\mathrm{i}}, \mathrm{i}=0, \ldots, N$

2. $T{ }_{N}^{\prime}\left(\mathrm{x}_{\mathrm{i}}\right)=0, \quad \mathrm{i}=1, \ldots, N-1$

3. $T_{N}^{\prime \prime}\left(\mathrm{x}_{\mathrm{i}}\right)=\frac{(-1)^{+1}}{1-x_{\mathrm{i}}^{2}} N^{2}, \quad \mathrm{i}=1, \ldots, N-1$

La aproximación de Chebyshev recurre a las fórmulas de la cuadratura Gaussiana, la cual aplicada a una cierta función $\mathrm{f}(\mathrm{x})$ conduce a la expresión

$$
\int_{-1}^{1} f(x) w(x) d x \cong \frac{\pi}{N} \sum_{i=0}^{N} \frac{f\left(x_{i}\right)}{\bar{c}_{i}}
$$

Donde

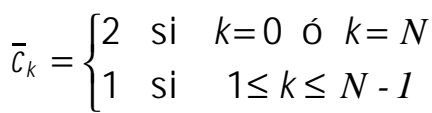

Si $f(x)$ es un polinomio de grado menor 0 igual a $2 \mathrm{~N}-1$, entonces la integral (30) es exacta. Ahora bien, como el producto $T_{k} T_{\text {l }}$ es un polinomio cuyo grado no excede a $2 \mathrm{~N}-1$, entonces de la ecuación (20) es posible deducir la relación de ortogonalidad discreta estimando la integral en cuestión con la fórmula de cuadratura (30) aplicada en los puntos de Gauss-Lobatto $x_{i}$, a saber:

$$
\sum_{i=0}^{N} \frac{1}{\overline{\mathrm{c}}_{\mathrm{i}}} T_{k}\left(\mathrm{x}_{\mathrm{i}}\right) T_{l}\left(\mathrm{x}_{\mathrm{i}}\right)=\frac{\overline{\mathrm{c}}}{2} N \delta_{k, l}
$$

Donde

$$
0 \leq k, I \leq N
$$

Evaluación de los coeficientes de Chebyshev

Para estimar los coeficientes a de la ecuación (24), mediante el método de colocación, se procede a anular el residual $R_{N}=u-u_{n}$ en ciertos puntos del dominio $\Omega$ elegidos de antemano, en el presente caso se opta por los puntos de Gauss-Lobatto, especificados ya por la ecuación (16).

Denotando $u_{i}=u\left(x_{i}\right)=u_{N}\left(x_{i}\right)$ y utilizando $T_{k}=$ $\cos \left(k \cos ^{-1} x\right)$, donde $x \in[-1,1]$ se deduce

$$
\mathrm{u}_{\mathrm{i}} \sum_{\mathrm{k}=0}^{N} \mathrm{a} \cos \frac{\mathrm{k} \pi \mathrm{i}}{N}, \quad \mathrm{i}=0,1 \ldots, N
$$

La expresión anterior, corresponde a un sistema algebraico de ecuaciones, siendo su matriz asociada

$$
T=\left[\frac{\cos k \pi \mathrm{i}}{N}\right], \quad \mathrm{k}, \mathrm{i}=0, \ldots, N
$$

esta matriz es invertible y mediante la relación de ortogonalidad discreta se deduce

$$
T^{-1}=\left[\frac{\frac{2(\cos \pi \mathrm{i})}{N}}{\left(\bar{c}_{k} \bar{c}_{i} N\right.}\right], \quad \mathrm{k}, \mathrm{i}=0, \ldots, N
$$

Luego, no es difícil verificar que la solución del sistema de ecuaciones algebraicas (33) resulta 


$$
\hat{\mathrm{u}}_{\mathrm{k}}=\frac{2}{\overline{\mathrm{c}}_{\mathrm{k}} N} \sum_{i=0}^{N} \frac{1}{\bar{c}_{i}} \mathrm{u}_{\mathrm{i}} \cos \frac{\mathrm{k} \pi \mathrm{i}}{N}, \mathrm{k}=0, \ldots, N
$$

\section{Diferenciación en el espacio físico}

Cuando se abordan problemas de valores en la frontera con el método de colocación, las incógnitas corresponden a valores de malla, y en consecuencia, se requiere expresar también a las derivadas en función de dichos valores.

Sea pues la matriz de diferenciación $\mathrm{D}$ definida por

$$
D=\left[d_{i, j}^{(\mathbb{D}}\right] \quad \mathrm{i}, \mathrm{j},=0, \ldots, N
$$

Luego las derivadas de primer y segundo orden se pueden expresar, respectivamente, por

$$
U^{(p)}=D^{(p)} U \text { con } p=1,2
$$

donde

$$
U^{(p)}=\left(u_{N}^{(p)}\left(x_{0}\right)_{,}, u_{n}^{(p)}\left(x_{n}^{(p)}\left(x_{N}\right)\right)\right.
$$

Aquí

$$
u_{N}^{(p)}\left(x_{i}\right)=\sum_{j=0}^{N} d_{i j}^{(p)} u_{N}\left(x_{j}\right), \quad p=12 \quad i=0, \ldots, N
$$

Si ahora se define la base de Lagrange $\left\{\left.\right|_{j}\right\}$ asociada a los puntos de colocación

$$
\mathrm{x}_{\mathrm{j}}=-\cos \theta_{\mathrm{j}}=-\cos \frac{\mathrm{j} \pi}{N}, 0 \leq \mathrm{j} \leq N,
$$

ubicados en el conjunto $\bar{I} \cup \partial i$ por

$$
\mathrm{I}_{\mathrm{j}}(\mathrm{x})=\frac{(-1)^{\mathrm{j}+1}\left(1-\mathrm{x}^{2}\right) T_{N}^{\prime}(\mathrm{x})}{\bar{c}_{j} N^{2}\left(\mathrm{x}-\mathrm{x}_{\mathrm{j}}\right)}
$$

entonces

$$
\mathrm{u}_{N}^{(\mathrm{p})}\left(\mathrm{x}_{\mathrm{i}}\right)=\sum_{j=0}^{N} \mathrm{~h}_{j}^{(\mathrm{p})} \mathrm{u}_{N}\left(\mathrm{x}_{\mathrm{j}}\right)
$$

Comparando las ecuaciones (40) y (42) se infiere que

$$
d_{i, j}^{(p)}=h_{j}^{(p)}\left(x_{i}\right)
$$

y además, no es difícil verificar que los términos de la matriz $D$ son

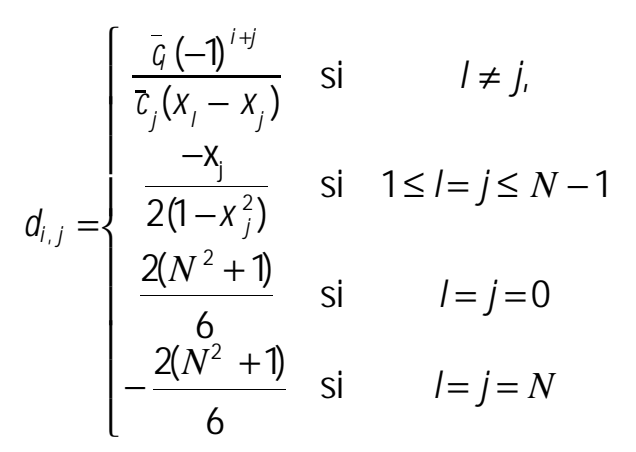

Finalmente, los términos de la matriz $D^{(2)}$ se pueden obtener mediante la siguiente ecuación

$$
d_{i, j}^{(2)}=\sum_{k=0}^{N} d_{i, k}^{(1)} d_{k, i}^{(1)}
$$

\section{Resultados numéricos}

\section{Introducción}

Para ilustrar la bondad de los métodos espectrales, se presentan a continuación dos ejemplos de aplicación a problemas de valores en la frontera.

Ambos casos fueron resueltos numéricamente, tanto con el método del elemento finito, como con un método espectral, con base en los polinomios de Chebyshev.

Los respectivos programas computacionales fueron instrumentados en la computadora Origin 2000 de la UNAM; la carga de trabajo quedó distribuida entre cuatro procesadores y se aprovecharon los recursos de la paquetería Sequent.

Los errores de las soluciones numéricas se presentan en las figuras 3 y 4 en escalas logarítmicas. 
> El eje de las abscisas corresponde al logaritmo en base 10 del número de nodos interiores de la malla ( $\log _{10}$ (N um.nodos)).

> El eje de las ordenadas corresponde al aditivo inverso del logaritmo en base 10 del error absoluto $\left(-\log _{10}(|u-a|)\right)$.

\section{Ejemplo de aplicación}

Se busca la solución numérica de la ecuación

$$
\frac{\partial^{2}}{\partial x^{2}} u(x, y)+\frac{\partial^{2}}{\partial y^{2}} u(x, y)=0
$$

en el dominio $\Omega=[0,1] \times[0,1]$, dadas las siguientes condiciones de frontera

$$
\left\{\begin{array}{cccc}
0 & \text { cuan do } & 0 \leq x \leq 1 & y=0 \\
\operatorname{sen} h(y) & \text { cuan do } & x=0 & 0 \leq y \leq 1 \\
\cos (x) \operatorname{sen} h(10) & \text { cuan do } & 0 \leq x \leq 1 & y=1 \\
\cos (10) \operatorname{sen} h(y) & \text { cuan do } & x=1 & 0 \leq y \leq 1
\end{array}\right.
$$

Se efectuaron 5 experimentos numéricos con pasos de malla

$$
h=\frac{1}{6}, h=\frac{1}{8}, h=\frac{1}{10}, h=\frac{1}{12}, h=\frac{1}{14}
$$

obteniéndose, respectivamente, 25, 49, 81, 121 y 225 nodos interiores.
Las aproximaciones numéricas obtenidas con los métodos del elemento finito y el método espectral, con base en los polinomios de Chebyshev, se contrastan con la solución exacta del problema, a saber,

$$
u(x, y)=\cos (x) \times \operatorname{sen} h(y)
$$

En la figura 3 se presenta la gráfica de los errores obtenidos.

\section{Ejemplo de aplicación 2}

Se busca la solución numérica de la ecuación

$$
\frac{\partial^{2}}{\partial x^{2}} u(x, y)+\frac{\partial^{2}}{\partial y^{2}} u(x, y)=0
$$

en el do minio $\Omega=[0,1] \times[0,1]$, dadas las siguientes condiciones de frontera

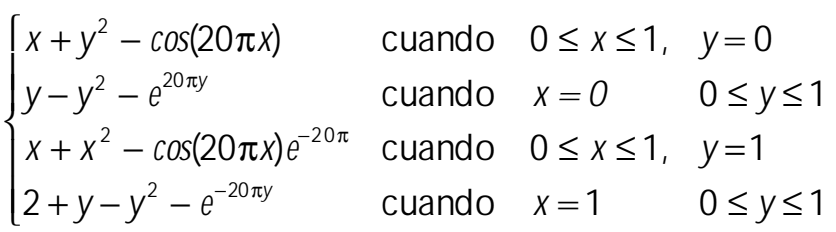

En este caso, la solución exacta del problema es

$$
x+y+x^{2}-y^{2}-\cos (20 \pi x) e^{-20 \pi y}
$$

Las aproximaciones numéricas obtenidas con los métodos del elemento finito y el método espectral con base en los polinomios de

- Método espectral

* Método del elemento finito

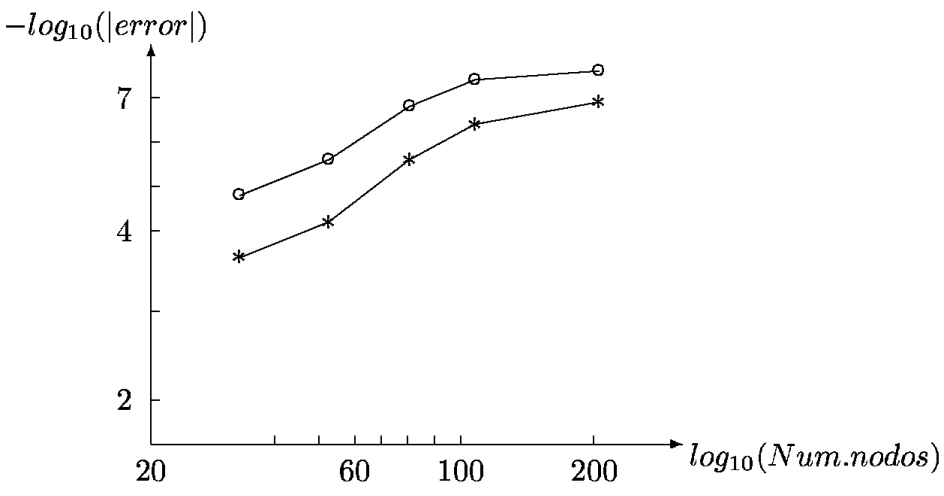

Figura 3. Errores de aproximación en la aplicación 1 


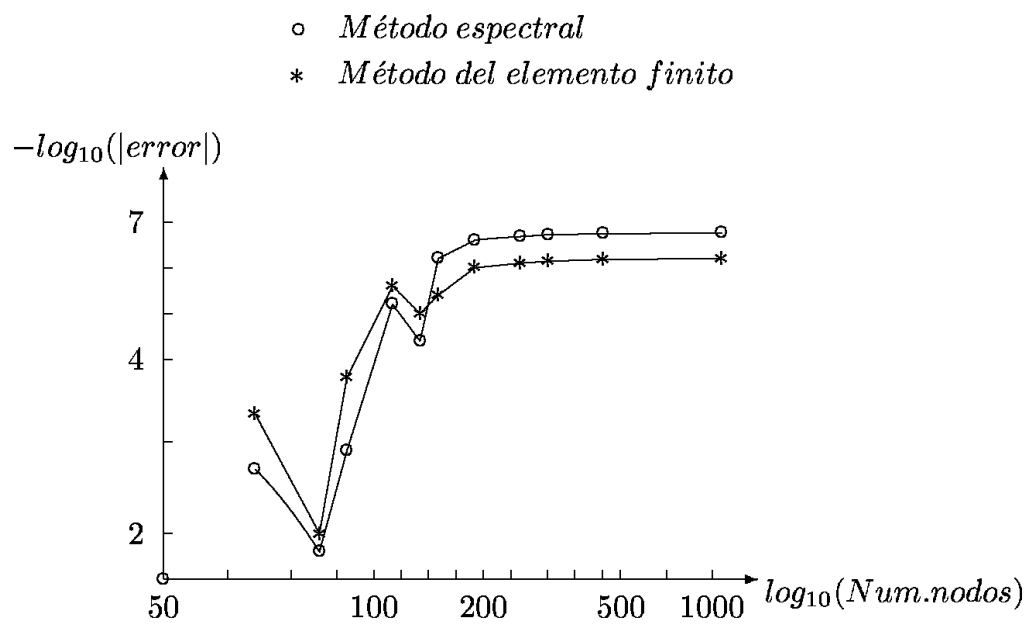

Figura 4. Errores de aproximación en la aplicación 2

Chebyshev, se contrastan con la solución exacta del problema.

En la figura 4 se presenta la gráfica de los errores obtenidos. En el presente ejemplo, el grado máximo de precisión se logró con un paso de malla $h=\frac{1}{34}$, a la cual le corresponden 1089 nodos interiores.

\section{Conclusiones}

La solución numérica de ambos ejemplos de aplicación, se obtuvo con la computadora Origin 2000 de la UNAM.

> Tanto los coeficientes de Chebyshev como los términos correspondientes a la diferenciación, se obtuvieron mediante el procesamiento paralelo con cuatro procesadores, aprovechando la paquetería Sequent.

> El sistema de ecuaciones lineales se resolvió también con cuatro procesadores recurriendo al paquete ScaLAPACK.

Como estos recursos, al presente sólo operan cálculos aritméticos con simple precisión, es así que las aproximaciones numéricas no exceden a ocho dígitos.

Los dos ejemplos de aplicación presentados, se eligieron con base en los siguientes criterios:

> Según el tipo de la solución del problema de valores en la frontera:
1. En el ejemplo de aplicación 1, la solución del problema se expresa mediante una función trascendente.

2. En el ejemplo de aplicación 2, la solución se expresa como la suma de una función trascendente y una función polinomial.

> Atendiendo al número máximo de oscilaciones o períodos que presenta la solución en el dominio:

1. En el ejemplo de aplicación 1 se obtuvo menos de un período.

2. En el ejemplo de aplicación 2 se obtuvo un número considerable de oscilaciones.

En ambos ejemplos de aplicación se verificó el excelente funcionamiento del método espectral con base en los polinomios de Chebyshev; en este punto procede mencionar que otro tanto sucedió en los experimentos numéricos desarrollados para el estudio de diversos problemas unidimensionales.

El ejemplo de aplicación 2 ilustra cómo en aquellos problemas de valores en la frontera donde la solución presenta un elevado número de oscilaciones, aun con mallas construidas con unas cuantas decenas o centenas de nodos, se obtienen excelentes resultados; para tal propósito, el paso de la malla h deberá ser de magnitud tal, que permita captar las posibles variaciones de la solución del problema en el dominio de su definición. En otras palabras, la naturaleza del error 
es semejante a la asociada a problemas de interpolación, donde la magnitud de aquél depende de la suavidad de la solución.

Aquí es obvia la utilidad de instrumentar en futuras etapas del presente tema algoritmos tipo $h-p$ que conjugen automáticamente la norma de la malla con el orden de los polinomios de Chebyshev.

\section{Agradecimientos}

Agradezco al M.I. Alberto Templos Carvajal por su apoyo y cooperación para el desarrollo de este artículo.

\section{Referencias}

Botella 0., Forestier M.Y., Pasquetti R., Peyret R. y Sabbah C. (2001a). Chebyshev Methods for the Navier-Stokes Equations: Algorithms and Applications. Nonlinear Anal, 47, pp. 4157-4168.

Botella 0. y Peyret R. (2001b). Computing Singular Solutions of the Navier-Stokes Equations with the Chebyshev-Collocation Method. Int. J. N umer. M ethods Fluids, 36, pp. 125-163.

Canuto C., Hussaini M.Y., Quarteroni A. y Zang T.A. (1988). Spectral Methods in Fluid Dynamics. Springer, New York.

Gottlieb D. y Orzag S.A. (1978). Numerical Analysis of Spectral Methods: Theory and Applications. SIAM - CBM S, Philadelfia.

Gottlieb D., Lustman L. y Tadmore E. (1987). Stability Analysis of Spectral Methods for Hyperbolic Initial-Boundary Value Problems. SIAM J. Numer. A nal, 24, pp. 241-258.

Gottlieb D. y Shu C.W. (1997). On the Gibbs Phenomenon and its Resesolution. SIAM Review, 39, pp. 644-668.

Guillard H., Malé J.M. y Peyret R. (1992). Adaptative Spectral Methods with Applications to Mixing Layer Computations. J. Comput. Phys, 102, pp. 379-388.

Kreiss 0. (1968). Stability Theory for Difference Approximations of Mixed Initial Boundary Value Problems I. Math. Comput, 22, pp. 703-714.

Mercier B. (1989). An Introduction to the Numerical A nalysis of Spectral M ethods. Springer Verlag, Berlin.
Orzag S.A. (1969). Numerical Methods for the Simulation of Turbulence. Phys. of Fluids, 12, (Suppl.II), pp. 250-257.

\section{Bibliografía sugerida}

Alencar H., Do Carmo M. y Marques F.C. (2001). Upper Bounds for the First Eigenvalue of Operator $L r$ and Some Applications. Illinois J. of Math. No. 45, pp. 851-863.

Arias J. (2000). Pointwise Convergence od Fourier Series. Springer Verlag, New York.

BLACS < http:// www.netlib.org/blacs/index. html>.

Bertola M., Eynard B. y Harnad J. (2002). Duality, Biorthogonal Polynomials and Multi-Matrix Models. Commun. M ath. Phys, 229, pp. 73-120.

Bertola M., Eynard B. y Harnad J. (2003). Differential Systems for Biorthogonal Polynomial Appearing in 2-Matrix Models and Associated Riemann-Hilbert Problems. Commun. Math. Phys, 243, pp. 193-240.

Forum MPI: A Message Passing Interface <ftp://www.netlib.org/mpi/mpi-report.ps>

Herrera I., Camacho A. y Hernández J. (1997). Domain Decomposition Methods for Model Parallelization. Proceedings of the Second UNAM-CRAY Supercomputing Conference, García F., Cisneros G., Fernández-Eguiarte A. y Álvarez R. (Eds), Cambridge University Press, pp. 258-265.

Herrera I., Hernández J., Camacho A. y Garfias J. (1997). Parallelization Using TH-Collocation. Proceedings of the Second UNAMCRAY Supercomputing Conference, García F., Cisneros G., Fernández-Eguiarte A. y Álvarez R. (Eds), Cambridge University Press, pp. 266-275.

Intel Corporation, Intel Supercomputer Technical Publications Home Page.

Langtangen H.P. (1997). Improving Efficiency of Diffpack Simulators for PDE's' Numerical Objects Report Series No. 1997:8, Numerical Objects AS, Oslo Noruega, < http:// www.nobjects.com/Reports/>. 
Langtangen H.P. (1997). Details of Finite Element Programming in Diffpack. Numerical Objects Report Series, No. 9, Numerical Objects AS, Oslo Noruega, < http:// www.nobjects.com/Reports/>.
Last Y. y Simon B. (1999). Eigenfunctions, Transfer Matrices and Absolutely

ScaLAPACK < http://www.netlib.org/scalapack /index.html>

Shubin M.A. (2001). PseudoD ifferential 0 perators and Spectral Theory. Springer Verlag, Berlin.

\section{Semblanza de los autores}

A bel Camacho- $G$ alván. Obtuvo las licenciaturas en ingeniería civil en la Universidad de Guadalajara y matemáticas en la Facultad de Ciencias de la UNAM, asimismo, las maestrías en ingeniería por parte de la UNAM y matemáticas por la UAM-Iztapalapa. Lo gró el doctorado en ciencias en el Instituto de Geofísica de la UNAM. Ha colaborado como académico e investigador en el Instituto de Ingeniería, el Instituto de Geofísica y en la Facultad de Ingeniería, todas instituciones pertenecientes a la UNAM, así como en el Instituto Mexicano del Petróleo, en UAM-Atzcapotzalco y UAM-Iztapalapa.

Beatriz Dolores Guardian-Soto. Obtuvo la licenciatura en computación por la UAM-Iztapalapa y la maestría en ciencias por el Instituto Politécnico Nacional. Es profesora investigadora de la misma institución en la ESIME-Cu, en el Departamento de Computación. Actualmente cumple con los créditos del doctorado en Ciencias Sociales, UAM- Xochimilco.

M ario Rodríguez-G reen. Cursó la licenciatura en matemáticas en la Facultad de Ciencias de la UNAM. Participó en proyectos de investigación en la Facultad de Ingeniería de la UNAM. Actualmente, colabora en el desarrollo de métodos de análisis numérico y su instrumentación computacional aplicados en la explotación de yacimientos petroleros. 